\title{
Protocol-Based Control for Cyber-Physical Systems with Environment-Dependent Energy Harvesting Sensors
}

\author{
Jiajia $\operatorname{Li}^{\mathrm{a}}$, Xin Tian ${ }^{\mathrm{a}, *}$, Guoliang $\mathrm{Wei}^{\mathrm{a}}$ \\ ${ }^{a}$ College of Science, University of Shanghai for Science and Technology, Shanghai 200093, P. R. China.
}

\begin{abstract}
In this paper, a protocol-based controller is designed for Cyber-Physical Systems (CPSs) with multiple sensors, which are powered by environment-dependent energy harvesting (EDEH) devices, respectively. The Round-Robin (RR) protocol is adopted to coordinate the data transmission of sensors. The protocol-based transmission can be realized only when the energy harvested by EDEH devices is sufficient. The aim of this paper is to design the protocol-based controller to ensure the stochastic finite-time boundedness (FTB) with EDEH and RR protocol. Firstly, modeling the EDEH by a switching sequence with varying sojourn probabilities, assuming a finite battery capacity constraint, and associating protocol-based transmission with a given energy cost, we propose a new recursive model to depict the dynamic of energy levels for each sensor. Then, combining with stochastic analysis and the dynamic of energy levels, the explicit expressions of the controller for each environment with average dwell time (ADT) are obtained. Finally, an example is provided to demonstrate the effectiveness of the designed controllers.
\end{abstract}

Keywords: Nonlinear systems, stochastic finite-time boundedness, environment-dependent energy harvesting, RR protocol.

\section{Introduction}

Due to the rapid development of computation, networking and communication, CPSs have attracted significant research interest $[1,2,3]$, which combine the cyber and physical process quite tightly. So far, CPSs have been applied in many fields, i.e. autonomous vehicles [4], industrial automation[5]. In these applications, a hot research topic is to study the control performance under the resources constraint (energy, bandwidth, computation and so on) [6]. In [7] and [8], the eventtriggering control scheme has been proposed for SCPs to decrease the data transmission burden with the limited communication resource. In [9], the resource allocation and control problem have been jointly studied for industrial CPSs under the power and subcarrier constraints.

As a vitally important branch of the resources constraint, energy problem often exists in CPSs $[10,11,12,13]$. In some applications of CPSs, the communication devices are battery powered vie a fixed power or a replaceable battery [14]. However, for some application scenarios, it is neither affordable nor feasible to always use a fixed supply or rechargeable battery, like Forest Fire

\footnotetext{
${ }^{*}$ Corresponding author

Email address: xytxmail@163.com (Xin Tian)
} 
Monitoring[15], oil spill remote sensing, Military environmental monitoring and so on. In the last decade, a new technology name Energy Harvest (EH) was presented in [16] and [17] to power the communication devices. EH technology can harvest energy from the environment, where the energy supply is potentially infinite but random and intermittent over time. In [18], EH process is assumed to be finite state Markov chains. In [19] and [20], the EH has been modeled by a two-state Markov chain ('Good' and 'Bad') and the Bernoulli distribution.

The EH devices is installed in fluctuating environments and environmental factors directly affect EH [21]. For the communication between the sensors and controllers under EDEH, the amount of energy harvested and stored is typically random and depends on the external environment. For example, solar panels only operate in sunlight and can collect energy with different probability distributions in different light conditions. As such, the energy analysis will be misleading if we use fixed statistical information, and our paper aims to provide a EDEH framework.

Moreover, to combat the scarcity of the communication resources, a series of communication protocol have been put forward. For example, as a periodic protocol, RR protocol has been proposed to dispatch the signals which are transmitted in a given circular function [22, 23]. RR protocol has got broad application in productions because of the structural simplicity and convenient implementation [24]. Consequently, an obvious problem is whether the stored energy under EDEH is insufficient to warrant the protocol-based transmission at certain time instants.

Based on the above discussions, we try to study protocol-based controllers to ensure the stochastic FTB of CPSs with EDEH sensors. The main contributions are: 1) Considering the phenomena of $\mathrm{EH}$ in environment-dependent modes, we make the first attempt to introduce the concept of EDEH modeled by environment-dependent Bernoulli distributions with known conditional probabilities. 2) A new recursive approach is proposed to calculate the probability of protocol-based energy storage level and information transmission under the EDDH. 3) Protocol-based controller is designed and the influence of the dwell time for each environment is analyzed under EDEH, where the technique is a recursive algorithm which is suitable for on-line computation.

The rest of this paper is organized as follows. Section II states preliminaries and problem formulation, followed by main results in Section III. In Section IV, an example is presented to demonstrate the effectiveness of the established design scheme. Finally, conclusions are drawn in Section V.

Notation: In this paper, $\lambda_{\max }(A)$ and $\lambda_{\min }(A)$ denote the maximum and minimum eigenvalue of $A$, respectively. $\mathbb{R}^{n}$ denotes the $n$ dimensional Euclidean space. The superscript " $T$ " stands for the transposition of considered matrices. The notation $P>0$ shows that $P$ is positive definite. $I$ denotes the identity matrix of compatible dimension and $\operatorname{diag}\{\cdot\}$ means a diagonal matrix.

\section{Problem Formulation}

As Figure 1 shows, the control process is equipped with EDEH sensors under the communication protocol, where the sensors harvest energy from different environments. The energy status and the communication protocols coordinatively determine the transmission of measurements to the remote controller. The system in Figure 1 is given as

$$
x(k+1)=A(k) x(k)+B(k) u(k)+D(k) w(k)+f(k, x(k), \psi(k))
$$

in a finite time horizon $k \in[1, N]$ with the $i$-th sensor's measurement output

$$
y_{i}(k)=C_{i}(k) x(k)+E_{i}(k) v(k)
$$




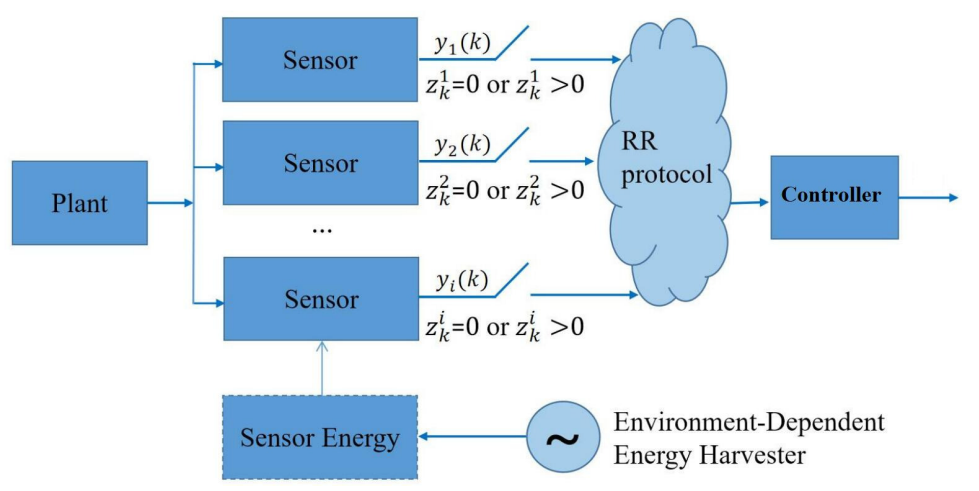

Figure 1: The control with EDEH sensors

where $x(k) \in \mathbb{R}^{n_{x}}$ represents the state variable, $u(k) \in \mathbb{R}^{n_{u}}$ and $y(k) \in \mathbb{R}^{n_{y}}$ denote the input and the output, $w(k) \in \mathbb{R}^{n_{x}}$ and $v(k) \in \mathbb{R}^{n_{y}}$ are the noise, which are unknown but satisfy

$$
\begin{aligned}
\mathcal{W}_{k} & =\left\{w^{T}(k) \bar{Q}(k) w(k)<\delta_{1}^{2}\right\}, \\
\mathcal{V}_{k} & =\left\{v^{T}(k) \tilde{Q}(k) v(k)<\delta_{2}^{2}\right\}
\end{aligned}
$$

where matrices $\bar{Q}(k)>0$ and $\tilde{Q}(k)>0 . A(k), B(k), C_{i}(k), D(k)$ and $E_{i}(k)$ are known time-varying matrices. $\psi(k)$ is a zero mean Gaussian noise sequence. The nonlinear item $f(k, x(k), \psi(k))$ satisfies

$$
\begin{aligned}
& \mathrm{E}\{f(k, x(k), \psi(k)) \mid x(k)\}=0, \\
& \mathrm{E}\left\{f(k, x(k), \psi(k)) f^{T}(j, x(j), \psi(j)) \mid x(k)\right\}=0, k \neq j \\
& \mathrm{E}\left\{f(k, x(k), \psi(k)) f^{T}(k, x(k), \psi(k)) \mid x(k)\right\}=\sum_{l=1}^{s} \pi_{l, k} \pi_{l, k}^{T} \mathrm{E}\left\{x^{T}(k) \Gamma_{l, k} x(k)\right\}
\end{aligned}
$$

where $s \in \mathbb{Z}^{+}, \pi_{l, k}$ and $\Gamma_{l, k}$ are known matrices.

The information exchange between sensors and controllers takes place over a communication network with limited resources. In the following, we will establish the measurement model under $\mathrm{RR}$ protocol and the environment-dependent energy harvesting.

\subsection{Round Robin Protocol}

In this paper, RR protocol is used to schedule the transmission, under which only one sensor can transmit information in turns. By defining $r(k)=\bmod \left(k-1, n_{y}\right)+1$ as the label of the active sensor, the measurement can be denoted as

$$
\tilde{y}(k)=\Phi_{r(k)} y(k)
$$

where $\Phi_{j}=\operatorname{diag}\left\{\delta(1-j), \delta(2-j), \ldots, \delta\left(n_{y}-j\right)\right\}, j=1,2, \ldots, n_{y}, \delta(\cdot) \in\{0,1\}$ is the Kronecker delta function. 


\subsection{Environment-dependent energy harvesting}

We denote the energy level for $i$-th sensor as $z_{k}^{i} \in\{0,1,2, \ldots, S\}$ where $S$ is the maximum energy level. The harvested energy at the $i$-th environment is represented by $h_{k}^{i}$ which is an environmentdependent independently identically distributed stochastic process with the probability distribution

$$
\begin{gathered}
\operatorname{Pr}\left(h_{k}^{i}=\gamma \mid \sigma_{k}=\beta\right)=p_{\gamma, \beta}^{i}, \\
\gamma=0,1,2,3 \ldots, \beta=1,2, \ldots, M
\end{gathered}
$$

where $\sigma_{k} \in\{1,2, \ldots, M\}$ is the environment factor, $p_{\gamma, \beta}^{i}$ satisfies $0 \leq p_{\gamma, \beta}^{i} \leq 1$ and $\sum_{\gamma=1}^{+\infty} p_{\gamma, \beta}^{i}=1$.

When $z_{k} \neq 0$, the sensor can transmit the measurement to the controller consuming 1 unit of energy. Thus, the indicator function and the measurement are further expressed by

$$
\eta_{k, \beta}^{i}=\left\{\begin{array}{lc}
1, & r(k)=i, z_{k}^{i} \geq 1, \sigma_{k}=\beta \\
0, & \text { else }
\end{array}\right.
$$

and $\hat{y}(k)=\left[\hat{y}_{1}(k), \hat{y}_{2}(k), \ldots, \hat{y}_{n_{y}}(k)\right]^{T}$ where $\hat{y}_{i}(k)=\eta_{k}^{i} y_{i}(k)+\left(1-\eta_{k}^{i}\right) \hat{y}_{i}(k-1)$. Moreover, the dynamics of the energy level for $i$-th sensor is

$$
z_{k+1}^{i}=\min \left\{z_{k}^{i}+h_{k+1}^{i}-\eta_{k}^{i}, S\right\}
$$

with $z_{0}^{i} \leq S$. Then, the controller can be described as

$$
u(k)=K_{\sigma_{k}}(k) \hat{y}(k)
$$

where $K_{\beta}(k)$ is the controller gain to be designed.

Consequently, denoting $\bar{x}(k)=\left[x^{T}(k), \hat{y}^{T}(k)\right]^{T}$ and $\bar{w}(k)=[w(k), v(k)]^{T}$, we have

$$
\begin{aligned}
\bar{x}(k+1)= & \sum_{\beta=1}^{M} \sigma_{\beta}(k)\left[\theta_{k, \beta}\left(\mathscr{A}_{1, \beta}(k) \bar{x}(k)+\mathscr{D}_{1, \beta}(k) \bar{w}(k)\right)+\left(I-\theta_{k, \beta}\right)\left(\mathscr{A}_{2, \beta}(k) \bar{x}(k)\right.\right. \\
& \left.\left.+\mathscr{D}_{2, \beta}(k) \bar{w}(k)\right)+\bar{f}(k, x(k), \psi(k))\right]
\end{aligned}
$$

where

$$
\begin{aligned}
& \mathscr{A}_{1, \beta}(k)=\left[\begin{array}{cc}
A(k)+B(k) K_{\beta}(k) C(k) & 0 \\
C(k) & 0
\end{array}\right], \mathscr{A}_{2, \beta}(k)=\left[\begin{array}{cc}
A(k) & B(k) K_{\beta}(k) \\
0 & I
\end{array}\right], \\
& \mathscr{D}_{1, \beta}(k)=\left[\begin{array}{cc}
D(k) & B(k) K_{\beta}(k) E(k) \\
0 & E(k)
\end{array}\right], \mathscr{D}_{2, \beta}(k)=\left[\begin{array}{cc}
D(k) & 0 \\
0 & 0
\end{array}\right], \\
& \theta_{k, \beta}=\operatorname{diag}\left\{\eta_{k, \beta}^{1}, \eta_{k, \beta}^{2}, \ldots, \eta_{k, \beta}^{m}, \eta_{k, \beta}^{1}, \eta_{k, \beta}^{2}, \ldots, \eta_{k, \beta}^{m}\right\}, C(k)=\left[C_{1}(k), C_{2}(k), \ldots, C_{m}(k)\right]^{T}, \\
& E(k)=\left[E_{1}(k), E_{2}(k), \ldots, E_{m}(k)\right]^{T}, \bar{f}(k, x(k), \psi(k))=[f(k, x(k), \psi(k)), 0]^{T},
\end{aligned}
$$

$\sigma_{\beta}(k)=1$ means that $\sigma_{k}=\beta$ and $\sum_{\beta=1}^{M} \sigma_{\beta}(k)=1$.

Given the stochastic variables $\alpha_{1}(k, \beta)=\eta_{k, \beta}^{r(k)}, \alpha_{2}(k, \beta)=1-\eta_{k, \beta}^{r(k)}$ and $\sigma_{\beta}(k)=\delta\left(\beta-\sigma_{k}\right)$ where $\delta(\cdot) \in\{0,1\}$ is the Kronecker delta function, we can rewrite the closed-loop nonlinear system as

$$
x(k+1)=\sum_{\beta=1}^{M} \sigma_{\beta}(k) \sum_{\mu=1}^{2} \alpha_{\mu}(k, \beta) \Theta_{k, \beta, \mu}\left[\mathscr{A}_{\mu, \beta}(k) \bar{x}(k)+\mathscr{D}_{\mu, \beta}(k) \bar{w}(k)+\bar{f}(k, x(k), \psi(k))\right]
$$

where

$$
\Theta_{k, \beta, \mu}=\left\{\begin{array}{cc}
\theta_{k, \beta}, & \mu=1 \\
I-\theta_{k, \beta}, & \mu=2
\end{array} .\right.
$$


Definition 1. [25] The discrete system with $w^{T}(k) \bar{Q}(k) w(k)<\delta_{1}^{2}, v^{T}(k) \tilde{Q}_{i}(k) v(k)<\delta_{2}^{2}$ is said to be stochastic FTB with $\left(c_{0}, c_{1}, \tau, R, \delta_{1}, \delta_{2}\right)$, if

$$
x^{T}(0) R x(0) \leq c_{0} \Rightarrow E\left\{x^{T}(k) R x(k)\right\}<c_{1}, k=1,2, \ldots, \tau
$$

where $c_{1}>c_{0}>0$ are real scalars and $R$ is a weight coefficient matrix.

The purpose is to design the controller such that system (4) stochastic FTB under RR protocol and EDEH.

\section{Main Results}

Firstly, we denote $\rho_{k, \beta}^{i}=\left[\operatorname{Pr}\left\{z_{k}^{i}=0 \mid \sigma_{k}=\beta\right\}, \operatorname{Pr}\left\{z_{k}^{i}=1 \mid \sigma_{k}=\beta\right\}, \ldots, \operatorname{Pr}\left\{z_{k}^{i}=S \mid \sigma_{k}=\beta\right\}\right]^{T}$ and

$$
\begin{aligned}
& \tilde{Q}_{\beta}^{i}=\left[\begin{array}{cccccc}
p_{0, \beta}^{i} & 0 & 0 & \cdots & 0 & 0 \\
p_{1, \beta}^{i} & p_{0, \beta}^{i} & 0 & \cdots & 0 & 0 \\
p_{2, \beta}^{i} & p_{1, \beta}^{i} & p_{0, \beta}^{i} & \cdots & 0 & 0 \\
\vdots & \vdots & \vdots & \ddots & \vdots & \vdots \\
p_{S-1, \beta}^{i} & p_{S-2, \beta}^{i} & p_{S-3, \beta}^{i} & \cdots & p_{0, \beta}^{i} & 0 \\
-\sum_{\mu=0}^{S-1} p_{\mu, \beta}^{i} & -\sum_{\mu=0}^{S-2} p_{\mu, \beta}^{i} & -\sum_{\mu=0}^{S-3} p_{\mu, \beta}^{i} & \cdots & -p_{0, \beta}^{i} & 0
\end{array}\right] \\
& \bar{Q}_{\beta}^{i}=\left[\begin{array}{cccccc}
p_{0, \beta}^{i} & p_{0, \beta}^{i} & 0 & 0 & \cdots & 0 \\
p_{1, \beta}^{i} & p_{1, \beta}^{i} & p_{0, \beta}^{i} & 0 & \cdots & 0 \\
p_{2, \beta}^{i} & p_{2, \beta}^{i} & p_{1, \beta}^{i} & p_{0, \beta}^{i} & \cdots & 0 \\
\vdots & \vdots & \vdots & \vdots & \ddots & \vdots \\
p_{S-1, \beta}^{i} & p_{S-1, \beta}^{i} & p_{S-2, \beta}^{i} & p_{S-3, \beta}^{i} & \cdots & p_{0, \beta}^{i} \\
-\sum_{\mu=0}^{S-1} p_{\mu, \beta}^{i} & -\sum_{\mu=0}^{S-1} p_{\mu, \beta}^{i} & -\sum_{\mu=0}^{S-2} p_{\mu, \beta}^{i} & -\sum_{\mu=0}^{S-3} p_{\mu, \beta}^{i} & \cdots & -p_{0, \beta}^{i}
\end{array}\right] .
\end{aligned}
$$

Lemma 1. For $\left\{z_{k}^{i}\right\}_{k \geq 0}$, the recursion of the probability $\rho_{k, \beta}^{i}$ under the $\beta$-th environment is given by

$$
\rho_{k+1, \beta}^{i}=[0,0, \ldots, 0,1]_{S+1}^{T}+\left[\eta_{k, \beta}^{i} \bar{Q}_{\beta}^{i}+\left(1-\eta_{k, \beta}^{i}\right) \tilde{Q}_{\beta}^{i}\right] \rho_{k,,}^{i},
$$

where $\rho_{0, \beta}^{i}=[\underbrace{0, \ldots, 0}_{z_{0}^{i}}, 1, \underbrace{0, \ldots, 0}_{S-z_{0}^{i}}]^{T}$ and $\beta=1,2, \ldots, M$.

Proof. According to $(2)$, the probability $z_{k}^{i}=t(0 \leq t<S)$ under the $\beta$-th environment can be denoted by

$$
\begin{aligned}
& \operatorname{Prob}\left\{z_{k+1}^{i}=t \mid \sigma_{k+1}=\beta\right\} \\
& =\operatorname{Prob}\left\{\min \left\{z_{k}^{i}+h_{k+1}^{i}-\eta_{k}^{i}, S\right\}=t \mid \sigma_{k+1}=\beta\right\} \\
& =\eta_{k}^{i} \operatorname{Prob}\left\{z_{k}^{i}+h_{k+1}^{i}-1=t \mid \sigma_{k+1}=\beta\right\}+\left(1-\eta_{k}^{i}\right) \operatorname{Prob}\left\{z_{k}^{i}+h_{k+1}^{i}=t \mid \sigma_{k+1}=\beta\right\} \\
& =\operatorname{Prob}\left\{z_{k}^{i}=0, h_{k+1}^{i}=t \mid \sigma_{k+1}=\beta\right\}+\eta_{k}^{i} \sum_{\mu=1}^{t+1} \operatorname{Prob}\left\{z_{k}^{i}=\mu, h_{k+1}^{i}=t+1-\mu \mid \sigma_{k+1}=\beta\right\}
\end{aligned}
$$




$$
\begin{aligned}
& +\left(1-\eta_{k}^{i}\right) \sum_{\mu=1}^{t} \operatorname{Prob}\left\{z_{k}^{i}=\mu, h_{k+1}^{i}=t-\mu \mid \sigma_{k+1}=\beta\right\} \\
= & \operatorname{Prob}\left\{z_{k}^{i}=0\right\} \operatorname{Prob}\left\{h_{k+1}^{i}=t \mid \sigma_{k+1}=\beta\right\}+\eta_{k}^{i} \sum_{\mu=1}^{t+1} \operatorname{Prob}\left\{z_{k}^{i}=\mu\right\} \operatorname{Prob}\left\{h_{k+1}^{i}=t+1-\mu \mid \sigma_{k+1}=\beta\right\} \\
& +\left(1-\eta_{k}^{i}\right) \sum_{\mu=1}^{t} \operatorname{Prob}\left\{z_{k}^{i}=\mu\right\} \operatorname{Prob}\left\{h_{k+1}^{i}=t-\mu \mid \sigma_{k+1}=\beta\right\} \\
= & p_{t, \beta}^{i} \operatorname{Prob}\left\{z_{k}^{i}=0\right\}+\eta_{k}^{i} \sum_{\mu=1}^{t+1} p_{t+1-\mu, \beta}^{i} \operatorname{Prob}\left\{z_{k}^{i}=\mu\right\}+\left(1-\eta_{k}^{i}\right) \sum_{\mu=1}^{t} p_{t-\mu, \beta}^{i} \operatorname{Prob}\left\{z_{k}^{i}=\mu\right\}
\end{aligned}
$$

Afterwards, the probability of $z_{k}^{i}=S$ can be expressed as

$$
\operatorname{Prob}\left\{z_{k+1}^{i}=S \mid \sigma_{k}=\beta\right\}=1-\sum_{\mu=0}^{S-1} \operatorname{Prob}\left\{z_{k+1}^{i}=\mu \mid \sigma_{k}=\beta\right\} .
$$

Combining (6) and (9), we can obtain the recursion (8). The proof is complete.

According to $\rho_{k, \beta}^{i}$ under the $\beta$-th environment, the transmission probability for $i$-th sensor can be obtained as $\varrho_{\beta}^{i}(k)=[0,1, \ldots, 1]_{S+1} \rho_{k, \beta}^{i}$. Then, the expectation of $\alpha_{1}(k, \beta)=\eta_{k, \beta}^{r(k)}=1$ under the $\beta$-th environment is $\bar{\alpha}_{1, k, \beta}=\varrho_{\beta}^{r(k)}(k)$ and the expectation of $\alpha_{2}(k, \beta)=1-\eta_{k, \beta}^{r(k)}=1$ is $\bar{\alpha}_{2, k, \beta}=1-\varrho_{\beta}^{r(k)}(k)$.

Now, we will study the corresponding control problems.

Theorem 1. Consider the closed-loop nonlinear system (4). Let the controller parameters $K_{\beta}(k)$, the parameters $c_{1}>0, c_{0}>0, \delta_{1}>0, \delta_{2}>0$ and matrices $P_{0}>0$ and $R>0$ be given. The expectation $\bar{\alpha}_{\mu, k, \beta}(\mu=1,2 ; \beta=1,2, \ldots, M)$ can be obtained by Lemma 1. System (4) is stochastic FTB with $\left(c_{0}, c_{1}, N, R, \delta_{1}, \delta_{2}\right)$ if there exist matrices $P_{k}>0, S>0$, nonsingular matrices $\Lambda(k)$ and scalars $\psi>1, \eta \geq 1, \lambda_{1}>0$ such that

1). for $k \in \mathbb{N}^{[1, N)}$

$$
\begin{gathered}
{\left[\begin{array}{cc}
\Omega_{t, k}^{11} & * \\
\Omega_{t, k}^{21} & \Omega_{t, k}^{22}
\end{array}\right]<0,} \\
{\left[\begin{array}{cc}
-P_{k+1}-I & \lambda_{1} P_{k+1}^{-1} R \\
* & -\lambda_{1} R
\end{array}\right]<0 ;}
\end{gathered}
$$

2). if $\sigma_{k}=\sigma_{k+1}$, then $P_{k+1}=P_{k}$ and $\eta_{k}=1$ hold, otherwise $P_{k+1}<\eta_{k} P_{k}$ and $\eta_{k}=\eta$ hold;

3). the inequality $\psi^{N}\left(\lambda_{2} c_{0}+\delta^{2} \lambda_{\max }(S)\right)<\lambda_{1} c_{1}$;

4). the ADT satisfies

$$
N_{a}>N_{a}^{*}=\frac{N \ln \eta}{\ln \kappa_{1}-\ln \kappa_{2}}
$$

where

$$
\Omega_{t, k}^{22}=\operatorname{diag}\left\{P_{k+1}-\Lambda(k)-\Lambda^{T}(k), P_{k+1}-\Lambda(k)-\Lambda^{T}(k)\right\},
$$




$$
\begin{aligned}
& \Omega_{t, k}^{11}= \operatorname{diag}\left\{\eta_{k} \sum_{l=1}^{s} \Gamma_{l, k} \operatorname{tr}\left(\pi_{l, k}^{T} P_{k} \pi_{l, k}\right)-\psi P_{k},-\psi S\right\}, \delta^{2}=N\left(\delta_{1}^{2}+\delta_{2}^{2}\right) / \min \left\{\lambda_{\min }(\bar{Q}), \lambda_{\min }(\tilde{Q})\right\}, \\
& \Omega_{t, k}^{21}= {\left[\sqrt{\bar{\alpha}_{1, k, \beta}}\left[\Theta_{k, \beta, \mu} \Lambda(k) \mathscr{A}_{1, \beta}(k), \Theta_{k, \beta, \mu} \Lambda(k) \mathscr{D}_{1, \beta}(k)\right]^{T},\right.} \\
&\left.\sqrt{\bar{\alpha}_{2, k, \beta}}\left[\Theta_{k, \beta, \mu} \Lambda(k) \mathscr{A}_{2, \beta}(k), \Theta_{k, \beta, \mu} \Lambda(k) \mathscr{D}_{2, \beta}(k)\right]^{T}\right]^{T}, \\
& \lambda_{2}=\lambda_{\max }\left(R^{-\frac{1}{2}} P_{0} R^{-\frac{1}{2}}\right), \kappa_{1}=(\eta-1) c_{1} \lambda_{1} \psi^{N}+\delta^{2} \lambda_{\max }(S) \text { and } \kappa_{2}=(\eta-1) c_{0} \lambda_{2}+\delta^{2} \lambda_{\max }(S) .
\end{aligned}
$$

Proof. For any time step $k(0<k \leq N)$ and the switching signal $\sigma_{k}$, let $0=k_{0}<k_{1}<\ldots<k_{j}<$ $\ldots<k(j=0,1,2, \ldots)$ denote the switching instants.

For $V(x(k), k)=x^{T}(k) P_{k} x(k)$ and the system (4) at $k \in \mathbb{N}^{\left[k_{j}, k_{j+1}\right)}$, we have

$$
\begin{aligned}
& \mathrm{E}\left\{x^{T}(k+1) P_{k+1} x(k+1)\right\} \\
= & \sum_{\beta=1}^{M} \sigma_{\beta}(k) \mathrm{E}\left\{\sigma_{\beta}(k) \sum_{\mu=1}^{2} \alpha_{\mu}(k, \beta) \Theta_{k, \beta, \mu}\left\{\mathscr{A}_{\mu, \beta}(k) \bar{x}(k)+\mathscr{D}_{\mu, \beta}(k) \bar{w}(k)+\bar{f}(k, x(k), \psi(k))\right\}^{T}\right. \\
& \left.\times P_{k+1}\left\{\mathscr{A}_{\mu, \beta}(k) \bar{x}(k)+\mathscr{D}_{\mu, \beta}(k) \bar{w}(k)+\bar{f}(k, x(k), \psi(k))\right\}\right\} \\
= & \sum_{\beta=1}^{M} \sigma_{\beta}(k)\left[\bar{x}^{T}(k), \bar{w}^{T}(k)\right] \Pi_{t, k}\left[\bar{x}^{T}(k), \bar{w}^{T}(k)\right]^{T}
\end{aligned}
$$

where

$$
\begin{aligned}
\Pi_{t, k} & =\sum_{\mu=1}^{2} \bar{\alpha}_{\mu, k, \beta}\left[\begin{array}{cc}
\Theta_{k, \beta, \mu} \Pi_{t, k}^{1} & * \\
\Theta_{k, \beta, \mu} \mathscr{A}_{\mu, \beta}^{T}(k) P_{k+1} \mathscr{D}_{\mu, \beta}(k) & \Theta_{k, \beta, \mu} \mathscr{D}_{\mu, \beta}^{T}(k) P_{k+1} \mathscr{D}_{\mu, \beta}(k)
\end{array}\right] \\
\Pi_{t, k}^{1} & =\mathscr{A}_{\mu, \beta}^{T}(k) P_{k+1} \mathscr{A}_{\mu, \beta}(k)+\sum_{l=1}^{s} \Gamma_{l, k} \operatorname{tr}\left(\pi_{l, k}^{T} P_{k} \pi_{l, k}\right) .
\end{aligned}
$$

Hence, one has

$$
\begin{aligned}
& \mathrm{E}\{V(x(k+1), k+1)\}-\psi \mathrm{E}\{V(x(k), k)\}-\psi \bar{w}^{T}(k) S \bar{w}(k) \\
= & \sum_{\beta=1}^{M} \sigma_{\beta}(k)\left[x^{T}(k), \bar{w}^{T}(k)\right] \Omega_{t, k}\left[x^{T}(k), \bar{w}^{T}(k)\right]^{T}
\end{aligned}
$$

where

$$
\Omega_{t, k}=\sum_{\mu=1}^{2} \bar{\alpha}_{\mu, k, \beta}\left[\begin{array}{cc}
\prod_{t, k}^{1}-\psi P_{k} & * \\
\Theta_{k, \beta, \mu} \mathscr{A}_{\mu, \beta}^{T}(k) P_{k+1} \mathscr{D}_{\mu, \beta}(k) & \Theta_{k, \beta, \mu} \mathscr{D}_{\mu, \beta}^{T}(k) P_{k+1} \mathscr{D}_{\mu, \beta}(k)-\psi S
\end{array}\right]
$$

Performing the congruence transformation $\operatorname{diag}\left\{I, I, \Lambda^{-T}(k), \Lambda^{-T}(k)\right\}$ to (8), then utilizing the inequality

$$
-P_{k+1}^{-1} \leq \Lambda^{-1}(k) P_{k+1} \Lambda^{-T}(k)-\Lambda^{-1}(k)-\Lambda^{-T}(k)
$$

the Schur complement lemma and condition (2), we get $\Omega_{t, k}<0$ and

$$
\mathrm{E}\{V(x(k+1), k+1)\}<\psi \mathrm{E}\{V(x(k), k)\}+\psi \bar{w}^{T}(k) S \bar{w}(k)
$$


for $k \in \mathbb{N}^{\left[k_{j}, k_{j+1}\right)}$.

Thus, for $\forall k \in \mathbb{N}^{\left[k_{j}, k_{j+1}\right)}$, we can obtain that

$$
\mathrm{E}\{V(x(k), k)\}<\psi^{k-k_{j}} \mathrm{E}\left\{V\left(x\left(k_{j}\right), k_{j}\right)\right\}+\sum_{s=k_{j}}^{k-1} \psi^{k-s} \bar{w}^{T}(s) S \bar{w}(s) .
$$

Combining $P_{k+1}<\eta P_{k}\left(\Pi_{k+1}^{*} \neq \Pi_{k}^{*}\right)$ and denoting $\tilde{P}_{k}=R^{-\frac{1}{2}} P_{k} R^{-\frac{1}{2}}$, we have

$$
\mathrm{E}\left\{V\left(x(k), k_{j+1}\right)\right\}<\eta \mathrm{E}\left\{V\left(x(k), k_{j}\right)\right\}
$$

and

$$
\begin{aligned}
\mathrm{E}\{V(x(k), k)\} & <\psi^{k-k_{0}}\left(\eta^{\frac{k-k_{0}}{N_{a}}} c_{0}\left(\lambda_{\max }\left(P_{0}\right)\right) x^{T}\left(k_{0}\right) x\left(k_{0}\right)+\frac{\eta^{\frac{k}{N_{a}}}-1}{\eta-1} \delta^{2} \lambda_{\max }(S)\right) \\
& =\psi^{k-k_{0}}\left(\eta^{\frac{k-k_{0}}{N_{a}}}\left(\lambda_{\max }\left(\tilde{P}_{0}\right)\right) x^{T}\left(k_{0}\right) R x\left(k_{0}\right)+\frac{\eta^{\frac{k}{N_{a}}}-1}{\eta-1} \delta^{2} \lambda_{\max }(S)\right) .
\end{aligned}
$$

Moreover, because of

$$
V(x(k), k)=x^{T}(k) R^{\frac{1}{2}} \tilde{P}_{k} R^{\frac{1}{2}} x(k) \geq \inf _{k \in[1, N]}\left(\lambda_{\min }\left(\tilde{P}_{k}\right)\right) x^{T}(k) R x(k),
$$

we have

$$
\mathrm{E}\left\{x^{T}(k) R x(k)\right\} \leq \frac{\psi^{k-k_{0}}}{\inf _{k \in[1, N]}\left(\lambda_{\min }\left(\tilde{P}_{k}\right)\right)}\left(\eta^{\frac{k-k_{0}}{N_{a}}} c_{0}\left(\lambda_{\max }\left(\tilde{P}_{0}\right)\right)+\frac{\eta^{\frac{k}{N_{a}}}-1}{\eta-1} \delta^{2} \lambda_{\max }(S)\right) .
$$

According to (9), we have $\tilde{P}_{k}>\lambda_{1} I$. Then, according to $\psi^{N}\left(\lambda_{2} c_{0}+\delta^{2} \lambda_{\max }(S)\right)<\lambda_{1} c_{1}$ and $N_{a}>N_{a}^{*}$, we obtain

$$
\mathrm{E}\left\{x^{T}(k) R x(k)\right\} \leq \frac{\psi^{k-k_{0}}}{\lambda_{1}}\left(\eta^{\frac{k-k_{0}}{N_{a}}} c_{0} \lambda_{2}+\frac{\eta^{\frac{k}{N_{a}}}-1}{\eta-1} \delta^{2} \lambda_{\max }(S)\right)=c_{1} .
$$

The proof is complete.

Theorem 2. For known parameters $c_{1}>0, c_{0}>0, \delta_{1}>0, \delta_{2}>0$, matrices $P_{0}>0, R>0$ and the expectation $\bar{\alpha}_{\mu, k, \beta}(\mu=1,2 ; \beta=1,2, \ldots, M)$ obtained by Lemma 1, system (4) is stochastic FTB with $\left(c_{0}, c_{1}, \tau, R, \delta_{1}, \delta_{2}\right)$ if exist $P_{k}>0, S>0, \Lambda(k), \bar{K}_{i}(k)(i=1,2, \ldots, N), \psi>1, \eta \geq 1, \mu_{k} \geq 1$ and $\lambda_{1}>0$ such that

$$
\left[\begin{array}{cc}
\Omega_{t, k}^{11} & * \\
\bar{\Omega}_{t, k}^{21} & \Omega_{k}^{22}
\end{array}\right]<0
$$

and b), c), d), e) in Theorem 1 hold where

$$
\begin{aligned}
& \bar{\Omega}_{t, k}^{21}=\left[\sqrt{\bar{\alpha}_{1, k, \beta}} \Theta_{k, \beta, 1} \hat{\Omega}_{t, k}^{1 T}, \sqrt{\bar{\alpha}_{2, k, \beta}} \Theta_{k, \beta, 2} \hat{\Omega}_{t, k}^{2 T}\right]^{T}, \hat{\Omega}_{t, k}^{j}=\left[\vec{\Omega}_{1}^{j}, \vec{\Omega}_{2}^{j}\right], j=1,2, \\
& \vec{\Omega}_{1}^{1}=\left[\begin{array}{cc}
\Lambda_{11}(k) W(k) A(k)+\bar{K}_{\beta}(k) C(k)+\Lambda_{12}(k) C(k) & 0 \\
\Lambda_{22}(k) C(k) & 0
\end{array}\right],
\end{aligned}
$$




$$
\begin{aligned}
& \vec{\Omega}_{2}^{1}=\left[\begin{array}{cc}
\Lambda_{11}(k) W(k) D(k) & \bar{K}_{\beta}(k) E(k)+\Lambda_{12}(k) E(k) \\
0 & \Lambda_{22}(k) E(k)
\end{array}\right], \\
& \vec{\Omega}_{1}^{2}=\left[\begin{array}{cc}
\Lambda_{11}(k) W(k) A(k) & \bar{K}_{\beta}(k)+\Lambda_{12} \\
0 & \Lambda_{22}
\end{array}\right], \\
& \vec{\Omega}_{2}^{2}=\left[\begin{array}{cc}
\Lambda_{11}(k) W(k) D(k) & 0 \\
0 & 0
\end{array}\right], \Lambda_{11}(k)=\left[\begin{array}{cc}
\tilde{\Lambda}_{11}(k) & \tilde{\Lambda}_{12}(k) \\
0 & \tilde{\Lambda}_{22}(k)
\end{array}\right], \\
& \bar{K}_{\beta}(k)=\left[\hat{K}_{\beta}^{T}(k), 0_{n_{u} \times\left(n_{y}-n_{x}\right)}\right]^{T}=\Lambda_{11}(k) W(k) B(k) K_{\beta}(k) \\
& \Lambda(k)=\left[\begin{array}{cc}
\Lambda_{11}(k) W(k) & \Lambda_{12}(k) \\
0 & \Lambda_{22}(k)
\end{array}\right], W(k)=\left[B(k)\left(B^{T}(k) B(k)\right)^{-1}\left(B^{T}(k)\right)^{\perp}\right]^{T}
\end{aligned}
$$

$\tilde{\Lambda}_{11}(k) \in \mathbb{R}^{n_{u} \times n_{u}}, \tilde{\Lambda}_{12}(k) \in \mathbb{R}^{n_{u} \times\left(n_{x}-n_{u}\right)}$ and $\tilde{\Lambda}_{22}(k) \in \mathbb{R}^{\left(n_{x}-n_{u}\right) \times\left(n_{x}-n_{u}\right)}$. Then, the controller gain is $K_{\beta}(k)=\tilde{\Lambda}_{11}^{-1}(k) \hat{K}_{\beta}(k)$.

Proof. Substituting Substituting $\mathscr{A}_{1, \beta}(k), \mathscr{A}_{2, \beta}(k), \mathscr{D}_{1, \beta}(k) \mathscr{D}_{2, \beta}(k)$ and $\bar{K}_{\beta}(k)=\Lambda_{11}(k) W(k) B(k) K_{\beta}(k)$ to Condition a) in Theorem 1, we have Condition a) is equivalent to (11).

Besides, it can be found from the structure of the matrix

$$
\begin{aligned}
\bar{K}_{\beta}(k) & =\Lambda_{11}(k) W(k) B(k) K_{\beta}(k)=\Lambda_{11}(k)[I, 0]^{T} K_{\beta}(k) \\
& =\left[\begin{array}{cc}
\tilde{\Lambda}_{11}(k) & \tilde{\Lambda}_{12}(k) \\
0 & \tilde{\Lambda}_{22}(k)
\end{array}\right]\left[\begin{array}{c}
K_{\beta}(k) \\
0
\end{array}\right] \\
& =\left[\left(\tilde{\Lambda}_{11}(k) K_{\beta}(k)\right)^{T}, 0\right]^{T}
\end{aligned}
$$

by plugging $\hat{K}_{\beta}(k)=\tilde{\Lambda}_{11}(k) K_{\beta}(k)$. Based on Theorem 1 , the desired controller can be obtained as $K_{\beta}(k)=\Lambda_{11}^{-1}(k) \hat{K}_{\beta}(k)$ by solving (11), which completes the proof.

According to Lemma 1 and the conditions in Theorem 2, the energy harvesting-dependent control algorithm can be concluded as follows.

\footnotetext{
Algorithm

Require: Set the recursive time $N$ and $k=0$. Initialize the environment factor $\sigma_{0} \in$ $\{1,2, \ldots, M\}$ and the energy level for $i$-th sensor $z_{0}^{i}\left(i=1,2, \ldots, n_{y}\right)$. Given the initial condition $x_{0}$, the transmit sequence $\{r(k)\}_{k=1,2, \ldots, N}$ under the Round-Robin protocol, the parameters $c_{1}>0, c_{0}>0, \delta_{1}>0, \delta_{2}>0$, matrices $P_{0}>0$ and $R>0$. Calculate the average dwell time $N_{a}$ according to the actual environment changes.

Step 1. Under the $\sigma_{k}$-th environment, obtain the expectations $\bar{\alpha}_{1, k, \sigma_{k}}$ and $\bar{\alpha}_{2, k, \sigma_{k}}$ according to Lemma 1. If the conditions in Theorem 2 is satisfied, calculate the control gain $K_{\sigma_{k}}(k)$ and the constraint $N_{a}^{*}$ of average dwell time, set $k=k+1$, and go to Step 2, else go to step 3 .

Step 2. If $k<N$, then go to Step 1, else go to Step 3.

Step 3. Stop.
} 


\section{Numerical simulation}

A numerical example is proposed in this section. For a nonlinear system with three sensors and the following parameters

$$
\begin{gathered}
A(k)=\left[\begin{array}{cc}
0.8+\cos (k) & 0.45 \\
0.3 & 0.6-\sin (k)
\end{array}\right], B_{1}(k)=\left[\begin{array}{ll}
1 & 0 \\
0 & 1
\end{array}\right], C(k)=\left[\begin{array}{lll}
1 & 0 & 0 \\
0 & 1 & 1
\end{array}\right]^{T}, \\
E(k)=[0.1,0.1,0.5]^{T}, D(k)=[0.4,0.2]^{T}, \pi=[0.2,0.3]^{T}, \Gamma=\operatorname{diag}\{0.01,0.16\}
\end{gathered}
$$

$c_{0}=0.1$ and $c_{1}=1.2$. The probabilities of energy harvesting at different environments are given in Table 1. The disturbance is $w_{i}(k)=v_{i}(k)=0.2 \sin (2 k)$, the initial state value is $x_{1}(0)=$ $[0.2,-0.2]^{T}, x_{2}(0)=[0.2,-0.1]^{T}, x_{3}(0)=[0.1,-0.2]^{T}$ and $\tau=50$. The nonlinear function is $f(k, x(k), \psi(k))=\left(0.1 \operatorname{sign}\left(x_{1}(k)\right) x_{1}(k) \psi_{1}(k)+0.4 \operatorname{sign}\left(x_{2}(k)\right) x_{2}(k) \psi_{2}(k)\right) \times[0.2,0.3]^{T}(i=1,2,3)$.

According to the given recursive algorithm, the controller gain can be obtained recursively by Matlab. Table 2 lists the desired parameters of controller $K_{1}(k)$ or $K_{2}(k)$ from the time $k=1$ to $k=12$. The bound is shown in Fig. 2. The switched environments are shown in Fig. 3. The energy level and measurement for $i$-th sensor are shown in Fig. 4-5. The simulation has confirmed that the designed controllers perform very well.

Table 1: the probabilities of energy harvesting

\begin{tabular}{|l|llll|lllll|llll|}
\hline Sensor & \multicolumn{3}{|c}{1} & & \multicolumn{3}{|c|}{2} & \multicolumn{5}{|c|}{3} \\
\hline Energy levels & 0 & 1 & 2 & 3 & 0 & 1 & 2 & 3 & 0 & 1 & 2 & 3 \\
\hline Environment 1 & 0.2 & 0.5 & 0.3 & 0 & 0.4 & 0.5 & 0.1 & 0 & 0.5 & 0.4 & 0.1 & 0 \\
\hline Environment 2 & 0.5 & 0.1 & 0.2 & 0.2 & 0.4 & 0.4 & 0.2 & 0 & 0.3 & 0.5 & 0.2 & 0 \\
\hline
\end{tabular}

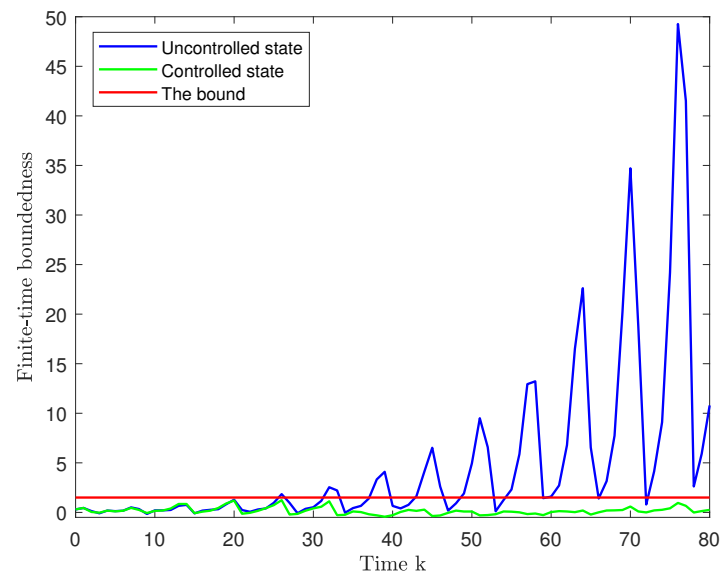

Figure 2: The open-loop system and the closed-loop system 
Table 2: the controller gain

\begin{tabular}{|c|c|c|c|c|c|c|}
\hline Time(mode) & \multicolumn{3}{|c|}{$1(1)$} & \multicolumn{3}{|c|}{$2(1)$} \\
\hline Control gain & $\begin{array}{l}-0.8353 \\
-0.2151\end{array}$ & $\begin{array}{c}-0.1801 \\
0.1043\end{array}$ & $\begin{array}{c}-0.1854 \\
0.1034\end{array}$ & $\begin{array}{l}-0.3569 \\
-0.2424\end{array}$ & $\begin{array}{c}-0.1414 \\
0.0750\end{array}$ & $\begin{array}{c}-0.1286 \\
0.0743\end{array}$ \\
\hline Time(mode) & \multicolumn{3}{|c|}{$3(1)$} & \multicolumn{3}{|c|}{$4(1)$} \\
\hline Control gain & $\begin{array}{c}0.1714 \\
-0.2819\end{array}$ & $\begin{array}{l}-0.2110 \\
-0.2362\end{array}$ & $\begin{array}{l}-0.1918 \\
-0.1696\end{array}$ & $\begin{array}{l}-0.0783 \\
-0.1666\end{array}$ & $\begin{array}{l}-0.1233 \\
-0.3636\end{array}$ & $\begin{array}{l}-0.0980 \\
-0.2949\end{array}$ \\
\hline $\begin{array}{l}\text { Time(mode) } \\
\text { Control gain }\end{array}$ & $\begin{array}{l}-0.3024 \\
-0.0352\end{array}$ & $\begin{array}{l}5(1) \\
-0.2885 \\
-0.6550\end{array}$ & $\begin{array}{l}-0.2377 \\
-0.5914\end{array}$ & $\begin{array}{l}-1.7098 \\
-0.2921\end{array}$ & $\begin{array}{l}6(1) \\
-0.3404 \\
-0.4847\end{array}$ & $\begin{array}{l}-0.1379 \\
-0.3967\end{array}$ \\
\hline Time(mode) & \multicolumn{3}{|c|}{$7(1)$} & \multicolumn{3}{|c|}{$8(1)$} \\
\hline Control gain & $\begin{array}{l}-0.5797 \\
-0.2337\end{array}$ & $\begin{array}{c}-0.1267 \\
0.1244 \\
\end{array}$ & $\begin{array}{c}-0.0913 \\
0.1333 \\
\end{array}$ & $\begin{array}{c}0.1110 \\
-0.2997 \\
\end{array}$ & $\begin{array}{l}-0.2344 \\
-0.1356 \\
\end{array}$ & $\begin{array}{l}-0.2146 \\
-0.0517 \\
\end{array}$ \\
\hline Time(mode) & \multicolumn{3}{|c|}{$9(2)$} & \multicolumn{3}{|c|}{$10(2)$} \\
\hline Control gain & $\begin{array}{l}-1.0773 \\
-0.2097 \\
\end{array}$ & $\begin{array}{l}-0.3393 \\
-0.0003 \\
\end{array}$ & $\begin{array}{c}-0.2351 \\
0.0150 \\
\end{array}$ & $\begin{array}{c}0.0329 \\
-0.2720 \\
\end{array}$ & $\begin{array}{l}-0.1716 \\
-0.4253 \\
\end{array}$ & $\begin{array}{l}-0.1563 \\
-0.3529 \\
\end{array}$ \\
\hline Time(mode) & \multicolumn{3}{|c|}{$11(2)$} & \multicolumn{3}{|c|}{$12(2)$} \\
\hline Control gain & $\begin{array}{l}-0.4618 \\
-0.1495 \\
\end{array}$ & $\begin{array}{l}-0.1626 \\
-0.5166 \\
\end{array}$ & $\begin{array}{l}-0.1284 \\
-0.4833\end{array}$ & $\begin{array}{l}-1.6429 \\
-0.2999 \\
\end{array}$ & $\begin{array}{l}-0.2381 \\
-0.5673 \\
\end{array}$ & $\begin{array}{l}-0.2124 \\
-0.5692 \\
\end{array}$ \\
\hline
\end{tabular}



Figure 3: The modes of the environment. 


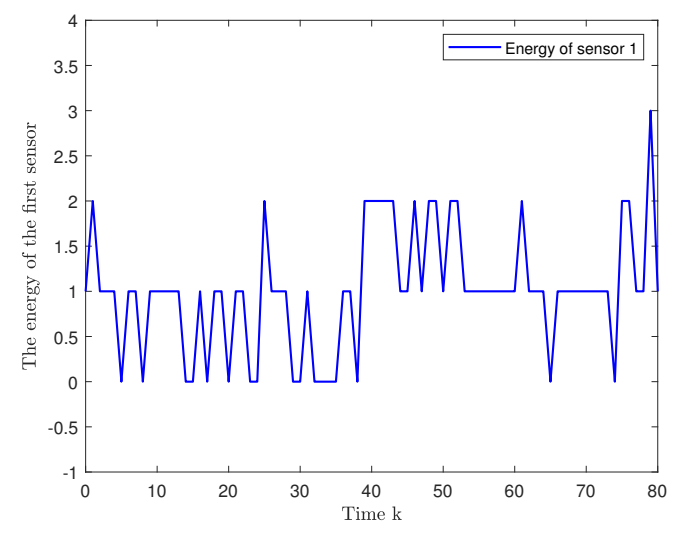

(a) The first sensor.

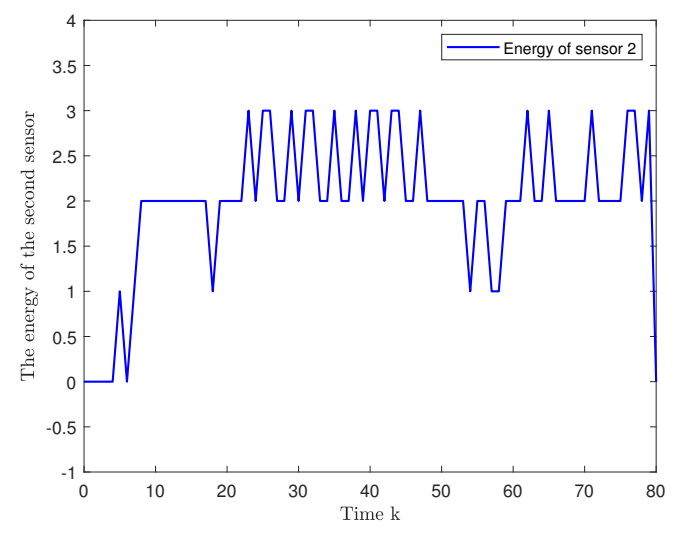

(b) The second sensor.

Figure 4: The energy.

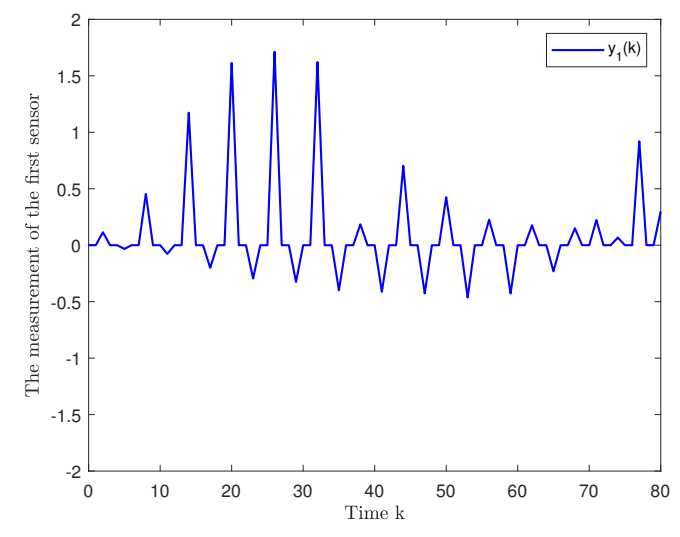

(a) The first sensor.

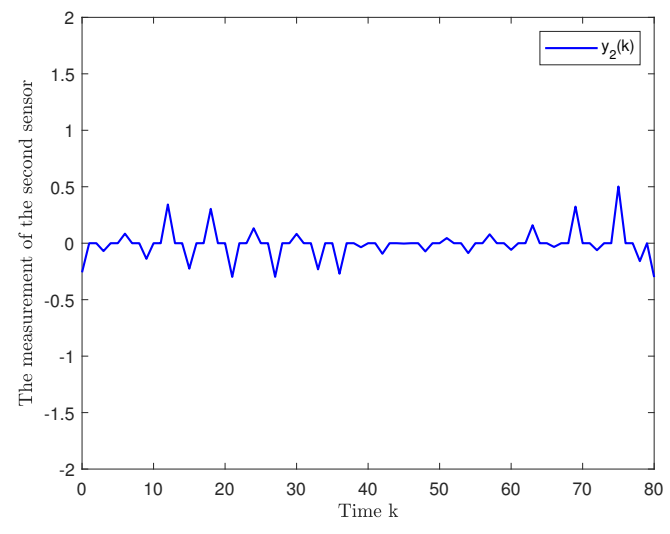

(b) The second sensor.

Figure 5: The measurement.

\section{Conclusions}

In this paper, the protocol-based control problem has been investigated for CPSs with EDEH devices. Firstly, an iterative algorithm of varying probabilities under EDEH and the RR protocol has been employed to describe the dynamics of the energy level for each sensor. Then, by utilizing ADT method and the varying probability of energy harvesting, the environment-dependent control problem case has been equivalently reduced to a general switched system with time-varying probability. A sufficient condition has been derived to guarantee the SFTB of the closed-loop system. 
Subsequently, the desired controller has been designed in terms of the proposed algorithm that can be adopted effectively by using available software. At last, an example has been given to proof the availability of the proposed design method.

\section{Acknowledgements}

This work was supported in part by the National Natural Science Foundation of China under Grants 61873169 and Shanghai Sailing Program 20YF1433200.

\section{Compliance with ethical standards}

\section{Conflict of interest}

The authors declare that they have no conflict of interest.

\section{References}

[1] Bhuiyan, M., Wu, J., Weiss, G., Hayajneh, T., Wang, T., Wang, G. J.: Event detection through differential pattern mining in cyber-physical systems. IEEE Transactions on Big Data. 6 (4), 652-665 (2020)

[2] Yan, J., Yang, X., Luo, X., Guan, X.: Dynamic gain control of teleoperating cyber-physical system with time-varying delay. Nonlinear Dynamics. 95 (4), 3049-3062 (2019)

[3] Yi, N., Xu, J. J., Yan, L. M., Huang, L.: Task optimization and scheduling of distributed cyber-physical system based on improved ant colony algorithm. Future Generation Computer Systems. 109, 134-148 (2020)

[4] Pasqualetti, F., Dörfler, F., Bullo, F.: Attack detection and identification in cyber-physical systems. IEEE Transactions on Automatic Control. 58 (11), 2715-2729 (2013)

[5] Jazdi, N.: Cyber physical systems in the context of industry 4.0, IEEE International Conference on Automation. 2014.

[6] Mamduhi, M., Maity, D., Baras, J., Johansson, K.: A cross-layer optimal co-design of control and networking in time-sensitive cyber-physical systems. IEEE Control Systems Letters. 5 (3), 917-922 (2021)

[7] Hamdan, M. M., Mahmoud, M. S., Baroudi, U. A.: Event-triggering control scheme for discrete time cyber physical systems in the presence of simultaneous hybrid stochastic attacks. ISA transactions (2021), doi:10.1016/j.isatra.2021.04.027.

[8] Sun, H. T., Peng, C., Xue, D., Wang, Y. L.: Resilient load frequency control of cyber-physical power systems under qos-dependent event-triggered communication. IEEE Transactions on Systems Man Cybernetics-Systems. 51 (4), 2113-2122 (2021)

[9] Termehchi, A., RastiJoint, M.: Joint sampling time and resource allocation for power efficiency in industrial cyber-physical systems. IEEE Transactions on Industrial Informatics. 17 (4), 2600-2610 (2021) 
[10] Deng, C., Wang, Y., Wen, C. Y., Xu, Y., Lin, P. F.: Distributed Resilient Control for Energy Storage Systems in Cyber-Physical Microgrids. IEEE Transactions on Industrial Informatics. $17(2), 1331-1341(2021)$

[11] Li, J., Wei, G., Ding, D., Tian, E.: Dwell-time-based energy scheduling and distributed control for large-scale nonlinear systems under round-robin protocol. Nonlinear Dynamics. $102(3), 1-14(2020)$

[12] Xie, G. Q., Zeng, G., Jiang, J. Q., Fan, C. N., Li, R. F., Li, K. Q.: Energy management for multiple real-time workflows on cyber-physical cloud systems. Future Generation Computer Systems. 105, 916-931 (2020)

[13] Zhou, Z. Y., Wang, B. C., Dong, M. X., Ota, K.: Secure and efficient vehicle-to-grid energy trading in cyber physical systems: integration of blockchain and edge computing. IEEE Transactions on Systems Man Cybernetics-Systems. 50 (1), 43-57 (2020)

[14] Niyato, D., Hossain, E., Rashid, M. M., Bhargava, V. K.: Wireless sensor networks with energy harvesting technologies: a game-theoretic approach to optimal energy management. IEEE Wireless Communications. 14 (4), 90-96 (2007)

[15] Boychuk, D., Braun, W. J., Kulperge, R. J., Krougly, Z. L., Stanford, D. A.: A stochastic forest fire growth model. Environmental and Ecological Statistics. 16 (2), 133-151 (2009)

[16] Calvo-Fullana, M., Antón-Haro, C., Matamoros, J., Ribeiro, A.: Random access communication for wireless control systems with energy harvesting sensors. IEEE Transactions on Signal Processing. 68, 3961-3975 (2020)

[17] Sharma, N., Mastronarde, N., Chakareski, J.: Delay-sensitive energy harvesting wireless sensors: Optimal scheduling, structural properties, and approximation analysis. IEEE Transactions on Communications. 68 (4), 2509-2524 (2020)

[18] Song, Y. B., Ye, D.: Optimizing DoS attack energy with imperfect acknowledgments and energy harvesting constraints in cyber-physical systems. Applied Mathematics and Computation. 393, no. 125821 (2021),.

[19] Michelusi, N., Stamatiou, K., Zorzi, M.: Transmission policies for energy harvesting sensors with time-correlated energy supply. IEEE Transactions on Communications. 61 (7), 2988-3001 (2013)

[20] Shen, B., Wang, Z. D., Wang, D., Luo, J., Pu, H. Y., Peng, Y.: Finite-horizon filtering for a class of nonlinear time-delayed systems with an energy harvesting sensor. Automatica. 100, 144-152 (2019)

[21] Zhang, Y., Ding, C., Wang, J., Cao, J.: High-energy orbit sliding mode control for nonlinear energy harvesting. Nonlinear Dynamics. 105(1), 191-211(2021)

[22] Shang, H., Zong, G.: Event-triggered sliding mode control under the round-robin protocol for networked switched systems. Nonlinear Dynamics. 100(6), 2401-2413 (2020)

[23] Chen, Y., Wang, Z. D., Wang, L. C., Shen, W. G.: finite-horizon $H_{\infty}$ state estimation for stochastic coupled networks with random inner couplings using round-robin protocol. IEEE Transactions on Cybernetics. 51 (3), 1204-1215 (2021) 
[24] Shen, B., Wang, Z. D., Wang, D., Liu, H. J.: Distributed state-saturated recursive filtering over sensor networks under Round Robin protocol. IEEE Transactions on Cybernetics. 50 (8), 3605-3615 (2020)

[25] Shen, H., Li, F., Wu, Z. G., Park, J. H.: Finite-time asynchronous $H_{\infty}$ filtering for discretetime markov jump systems over a lossy network. International Journal of Robust and Nonlinear Control 26 (17), 3831-3848 (2016) 